\title{
Parasitas intestinais em crianças de escola municipal de Florianópolis, SC - Educação ambiental e em saúde
}

\author{
Jaques Muriel Oliveira Kunz ${ }^{1}$ \\ Abel Silva Vieira ${ }^{1}$ \\ Theopi Varvakis ${ }^{1}$ \\ Guilherme Araújo Gomes ${ }^{1}$ \\ Ana Letícia Rossetto ${ }^{1}$ \\ Orlando José Bernardini ${ }^{1}$ \\ Marilia Sirianni dos Santos Almeida ${ }^{2 *}$ \\ Maria Márcia Imenes Ishida ${ }^{1}$
${ }^{1}$ Departamento de Microbiologia e Parasitologia, Centro de Ciências Biológicas
Universidade Federal de Santa Catarina, Florianópolis - SC, Brasil
${ }^{2}$ Instituto Osvaldo Cruz, Rio de Janeiro - RJ, Brasil
*Autor para correspondência
sirianni@ccb.ufsc.br

Submetido em 08/11/2007

Aceito para publicação em 13/06/2008

\section{Resumo}

As parasitoses intestinais constituem sério problema de saúde pública no Brasil, apresentando maior prevalência em populações de nível sócio-econômico mais baixo e com condições precárias de saneamento básico. O objetivo do trabalho foi avaliar a prevalência de enteroparasitoses em crianças que freqüentam a Escola de Educação Básica Intendente Aricomedes da Silva, da rede municipal de ensino, situada no bairro Cachoeira do Bom Jesus em Florianópolis (SC), e desenvolver atividades com as crianças e comunidade visando à prevenção de parasitoses. Durante os meses de junho a dezembro de 2006 foram analisadas amostras fecais de 101 escolares e 5 adultos, das quais 35,8\% (38) estavam parasitadas. Os protozoários mais freqüentes foram Entamoeba coli (20,7\%) e Endolimax nana (12,3\%) que, embora não patogênicos, indicam contaminação por via fecal-oral. Entre os helmintos, o Ascaris lumbricoides teve maior prevalência (5,7\%). Os indivíduos positivos foram encaminhados ao Posto de Saúde onde receberam tratamento adequado. Realizou-se aula teórica e saída de campo com as crianças, e palestra com a comunidade escolar e pais dos alunos, visando à educação em saúde e meio ambiente.

Unitermos: enteroparasitoses, saneamento básico, escolares, Florianópolis 


\section{Abstract}

Intestinal parasite infection in children from primary school in Florianopolis (SC) - Environmental and health education. Parasite infection remains an important public health problem in many areas around the world as well as in Brazil, and it is frequently associated with poverty and lack of sanitation facilities. A coprological investigation was conducted in children from the primary school Intendente Aricomedes da Silva in Florianopolis, Brazil, in order to determine the prevalence of intestinal parasite infections. Also a series of indoor and outdoor activities were carried out to improve the awareness of students, parents, and school staff about parasite infection. Fecal samples from 101 school children and 5 school adult staff were collected and analyzed from June to December 2006. Thirty-eight individuals (35.8\%) were positive for at least one parasite. Ascaris lumbricoides, the most frequent helminth, was prevalent in $5.7 \%$ of individuals. Entamoeba coli and Endolimax nana were the most prevalent protozoa in this study: $20.7 \%$ and $12.3 \%$ respectively. Although non pathogenic protozoa species, they indicate oral-fecal contamination. Infected individuals were sent to the Health Unit for treatment. Finally, a meeting with the school community was organized to discuss how to prevent intestinal parasite infections by improving basic hygiene habits and best practice with water, food and environment.

Key words: enteroparasitosis, health education, school children, Florianópolis

\section{Introdução}

As parasitoses intestinais constituem-se em grave problema de saúde pública especialmente nos países em desenvolvimento onde são endêmicas, contribuindo para problemas econômicos e sociais (Silva e Santos, 2001; UNEP, 2006). No Brasil, as enteroparasitoses são freqüentes, especialmente entre as crianças e as principais conseqüências são: diarréia crônica, máabsorção, anemia ferropriva, baixa capacidade de concentração e dificuldades no aprendizado.

A prevalência das enteroparasitoses, cuja transmissão se dá pela via fecal-oral ou penetração pela pele, é maior nas áreas de baixas condições sócioeconômicas e carentes de saneamento básico, incluindose o tratamento de água, esgoto, recolhimento do lixo e o controle de vetores (Castro et al., 2004; Ferreira et al., 2004; Basualdo et al. 2007; Teixeira, et al., 2007).

O que mais dificulta a implementação de ações de controle, além do custo financeiro e das medidas técnicas, é a falta de projetos de educação sanitária com a integração da comunidade (Silva e Santos, 2001; Abraham et al., 2007).

Florianópolis, capital que sofre como os demais municípios catarinenses, seja pela ausência ou precariedade de saneamento básico, seja por questões que envolvam os cuidados com higiene individual ou de instalações de reservatórios para água, atualmente tem seu quadro agravado pela explosão demográfica, não acompanhada de investimentos em infraestrutura de saneamento e saúde, refletindo diretamente nas condições ambientais da cidade.

No Norte da Ilha de Santa Catarina, somente as praias de Canasvieiras, Jurerê Internacional e Brava possuem estação de tratamento de esgoto. Nas demais, o tratamento é feito pelo sistema de fossas que freqüentemente é mal planejado e, sendo ligado diretamente ao sistema de águas pluviais, afeta a balneabilidade das praias.

Nas praias da Cachoeira do Bom Jesus e Ponta das Canas, situadas ao Norte da Ilha de Santa Catarina, não existe sistema de coleta de esgoto e em diferentes áreas dos balneários é visível a chegada de esgoto doméstico ao meio-ambiente, o que fez com que no final do ano de 2006 a Fundação do Meio Ambiente de Santa Catarina - FATMA considerasse a maioria dos pontos avaliados na região impróprios para banho (FATMA, 2006).

Dentro deste contexto, o presente estudo visou investigar a ocorrência de parasitoses intestinais através de exames coproparasitológicos em adultos e escolares de $1^{\mathrm{a}}$ a $4^{\mathrm{a}}$ séries de escola municipal objetivando a implementação de projetos educativos com a participação da comunidade nas ações de prevenção e controle dos agravos à saúde e ao meio ambiente. 


\section{Material e Métodos}

O presente trabalho foi realizado, no período de junho a dezembro de 2006, na Escola Intendente Aricomedes da Silva, da rede municipal de ensino, situada no bairro Cachoeira do Bom Jesus em Florianópolis (SC).

O estudo foi submetido ao Comitê de Ética em Pesquisa com Seres Humanos da Universidade Federal de Santa Catarina (UFSC) obtendo parecer favorável sob registro de $n^{\circ} .362 / 06$.

Os dados foram submetidos ao teste qui-quadrado, considerando-se diferenças significativas, valores de $\mathrm{p}<0,05$.

\section{Reuniões preparatórias}

Inicialmente, foram feitas reuniões preparatórias com a direção da escola e posteriormente, com professores e pais dos alunos para apresentação dos propósitos da investigação.

\section{Coleta de amostras}

Para a coleta de amostras foram distribuídos 277 coletores universais de fezes para alunos de $1^{\text {a }}$ a $4^{\mathrm{a}}$ séries $(\mathrm{n}=251)$ e funcionários da escola $(\mathrm{n}=26)$, tendo sido devolvidos 106 coletores com amostras (101 de escolares e 5 de funcionários, 51 do sexo masculino e 55 do sexo feminino). Juntamente com os coletores foram entregues os termos de consentimento livre e esclarecido (TCLE), para a assinatura dos participantes ou de pais/responsáveis de menores de 18 anos, e também um informativo com instruções e cuidados a serem observados durante a coleta do material.

As amostras foram acondicionadas em refrigerador disponibilizado na escola para esta finalidade e, posteriormente transportadas para o Laboratório de Helmintologia do Departamento de Microbiologia e Parasitologia (MIP) da UFSC para realização dos exames parasitológicos.

\section{Métodos parasitológicos}

Foram empregados os métodos de sedimentação de Hoffman et al. (1934) e de centrifugo-flutuação de
Faust et al. (1939). Para cada amostra foram preparadas quatro lâminas (duas para cada método) examinadas por dois observadores em microscopia óptica com aumentos de $100 \mathrm{X}$ e $400 \mathrm{X}$.

\section{Atividades com os escolares}

Com base nos resultados dos exames, foram feitas atividades com os alunos sobre a problemática das parasitoses intestinais na região e sua ligação com as questões ambientais, incluindo saídas de campo no entorno da escola para averiguação das condições sanitárias, presença de efluentes nos rios e manguezal, além de atividades em sala como representação em desenhos das situações observadas em campo.

\section{Resultados}

Das 106 amostras analisadas, 38 (35,8\%) apresentaram uma ou mais espécies de parasita e/ou comensal (Tabela 1).

TABELA 1: Resultados de exames coproparasitológicos de membros da Escola Municipal Intendente Aricomedes da Silva, Cachoeira do Bom Jesus, Florianópolis-SC, 2006.

\begin{tabular}{l|c|c|c}
\multicolumn{1}{c|}{ Amostras } & Ocorrência & $\begin{array}{c}\text { \% do total } \\
(\boldsymbol{n = 1 0 6})\end{array}$ & $\begin{array}{c}\text { \% entre } \\
\text { positivos }\end{array}$ \\
\hline Positivas & 38 & 35,8 & 100 \\
\hline Um parasita & 26 & 24,5 & 68,4 \\
\hline $\begin{array}{l}\text { Dois ou mais pa- } \\
\text { rasitas }\end{array}$ & 12 & 11,3 & 31,6 \\
\hline Negativas & 68 & 64,1 & - \\
\hline
\end{tabular}

Dos 12 casos de poliparasitismo encontrados, três apresentaram tripla associação de parasitas e os demais, dupla associação.

Das 38 pessoas infectadas com ao menos um parasita, $18(32,7 \%)$ eram do sexo feminino $(n=55)$ e $20(39,2 \%)$ do sexo masculino $(\mathrm{n}=51)$, não havendo diferença estatística significativa ( $\mathrm{p}>0,05)$.

Os parasitas encontrados são apresentados na Tabela 2. 
TABELA2: Espécies de parasitas intestinais encontradas em membros da Escola Intendente Aricomedes da Silva, Cachoeira do Bom Jesus, Florianópolis-SC, 2006.

\begin{tabular}{l|c|c|c}
\multicolumn{1}{c|}{ Parasita } & Ocorrência & $\begin{array}{c}\text { \% do total } \\
(\boldsymbol{n = 1 0 6 )}\end{array}$ & $\begin{array}{c}\text { \% entre } \\
\text { positivos } \\
(\boldsymbol{n}=\mathbf{3 8})\end{array}$ \\
\hline Entamoeba coli & 22 & 20,7 & 57,9 \\
\hline Endolimax nana & 13 & 12,3 & 34,2 \\
\hline Ascaris lumbricoides & 6 & 5,7 & 15,8 \\
\hline Giardia lamblia & 5 & 4,7 & 13,8 \\
\hline Trichuris trichiura & 3 & 2,8 & 7,9 \\
\hline Hymenolepis nana & 2 & 1,9 & 5,3 \\
\hline $\begin{array}{l}\text { Enterobius vermicu- } \\
\text { laris }\end{array}$ & 1 & 0,9 & 2,6 \\
\hline
\end{tabular}

Dos cinco adultos examinados, três tiveram resultado positivo, sendo um (albergando E. coli) funcionário da cozinha da escola.

\section{Entrega de resultados e tratamento}

Os resultados dos exames foram encaminhados à direção da escola que os entregou aos adultos e aos pais dos escolares. Os indivíduos positivos foram encaminhados ao Posto de Saúde da Cachoeira do Bom Jesus onde receberam tratamento adequado.

\section{Palestras com a comunidade}

Inicialmente, foi realizada uma reunião com a comunidade escolar e pais de alunos, e em outra ocasião com a AMANGUE (Associação dos Amigos do Mangue da Cachoeira do Bom Jesus), onde foram apresentados resultados do presente trabalho e de trabalho anterior, que evidenciou a presença de parasitas humanos nos corpos de água do manguezal daquela localidade (Vieira et al., 2005), dando ênfase aos principais aspectos do ciclo biológico dos parasitas intestinais humanos e seus mecanismos de transmissão. Foram discutidos os fatores ambientais que contribuem para a disseminação de parasitoses e medidas profiláticas e de controle, com finalidade de conscientização da importância da participação nas ações de interesse da comunidade.

\section{Discussão}

A baixa adesão de crianças à etapa de coleta e entrega de amostras de fezes foi inesperada face à motivação demonstrada nas demais atividades, porém a dependência do auxilio dos pais para a coleta das amostras pode ter dificultado a tarefa.

A freqüência encontrada de parasitoses intestinais nos escolares da Cachoeira do Bom Jesus (35,5\%) revelou-se relativamente baixa quando comparada a estudos feitos em outras cidades do Brasil com alunos da rede pública: Saturnino et al. (2003) avaliaram em 1999 um total de 100 crianças entre um e cinco anos, residentes no bairro de Cidade Nova, em Natal (RN) e a prevalência geral encontrada para parasitismo intestinal foi de $76 \%$, sendo os parasitas mais freqüentes Ascaris lumbricoides (41,7\%), Trichuris trichiura (40,5\%), Ancilostomidae $(8,3 \%)$, Giardia lamblia (20,7\%) e Entamoeba histolytica (15,3\%). Castro et al. (2004), no período de março a novembro 2003, analisaram 421 amostras fecais de escolares da rede pública de Cachoeiro de Itapemirim (ES), na faixa etária de 8 a 15 anos e encontraram o índice de 19,7\% de parasitoses intestinais, sendo Giardia lamblia (34,9\%), Entamoeba coli (22,9\%), Endolimax nana $(9,6 \%)$ e Ascaris lumbricoides (4,8\%) os parasitas mais freqüentes. Ferreira et al. (2003) evidenciaram $59,7 \%$ de positividade em escolares de Campo Florido (MG). Quadros et al. (2004) encontraram em crianças de Lages (SC) uma prevalência total de 70,5\% e Buschini et al (2007) mostraram que $75,3 \%$ das 635 crianças de sete comunidades escolares do município de Guarapuava (PR) apresentaram parasitas intestinais, sendo o mais freqüente, Giardia duodenalis (56\%) e em segundo lugar, Ascaris lumbricoides (18\%).

As precárias condições de saneamento básico expõem as populações à aquisição de diferentes patógenos, tornando freqüentes os casos de poliparasitismo. Castro et al. (2004) e Buschini et al (2007) citados acima, encontraram em comunidades escolares de diferentes regiões do país, respectivamente, $10,8 \%$ e $26,7 \%$ de associações de parasitas.

No presente estudo, encontramos 12 casos de associações $(11,3 \%)$ (Tabela 1), que embora não representem uma alta freqüência, indicam exposição dos 
indivíduos a diferentes patógenos - todos, neste caso, adquiridos pela mesma via de infecção. Em trabalho anterior, de investigação de enteroparasitoses humanas em amostras de água coletadas no manguezal da região (Vieira et al. 2005), foram encontradas as mesmas espécies parasitárias diagnosticadas nos escolares do presente estudo, sugerindo a ocorrência do ciclo destes parasitas neste ambiente.

Nenhuma diferença significativa na positividade entre indivíduos de diferentes idades e sexo foi observada quando os dados foram submetidos ao teste do Qui-quadrado.

A baixa taxa de positividade de parasitoses intestinais encontrada entre os examinados no presente trabalho não elimina a necessidade de adoção de medidas de controle e erradicação da transmissão.

A presença dos protozoários intestinais comensais E. coli e E. nana não constitui agravo à saúde, porém indica contaminação por via fecal-oral, estando os sujeitos suscetíveis à aquisição de patógenos. Um dos indivíduos adultos com resultado positivo para protozoários de transmissão fecal-oral é um funcionário da cozinha da escola. Segundo Nolla e Cantos (2005), na cidade de Florianópolis é alta a ocorrência de parasitoses entre manipuladores de alimentos, o que requer aprimoramento na educação sanitária e maior controle epidemiológico sobre esses profissionais.

$\mathrm{O}$ tratamento dos indivíduos parasitados, sem que sejam conhecidas e extintas as fontes de contaminação, pode se constituir em medida apenas paliativa. Dados da literatura mostram que a combinação de medidas como saneamento e educação sanitária são as opções de escolha para minorar as infecções parasitárias (Bóia et al. 2006).

O presente trabalho serve de indicador das condições de saneamento em que vivem as crianças do bairro Cachoeira do Bom Jesus, possibilitando nova postura da comunidade na solução dos problemas sócio-ambientais. O trabalho contribui ainda para o conhecimento das parasitoses intestinais encontradas na localidade estudada, tendo em vista a inexistência de dados anteriores.

\section{Referências}

Abraham, R. de S.; Tashima, N. T.; Silva, M. A. da 2007. Prevalência de enteroparasitoses em reeducandos da Penitenciária "Maurício Henrique Guimarães Pereira" de Presidente Venceslau - SP. Revista Brasileira de Análises Clinicas, 39 (1): 39-42.

Basualdo, J. A.; Córdoba, M. C.; De Luca, M.; Ciarmela, M. L.; Pezzani, B. C.; Grenovero, M. S.; Minvielle, M. C. 2007. Intestinal parasitoses and environmental factors in a rural population of Argentina, 2002-2003. Revista do Instituto de Medicina Tropical de São Paulo, 49 (4): 251-255.

Bóia, M. N.; Carvalho- Costa F. A.; Sodré, F. C.; Eyer-Silva, W. A.; Lamas, C. C.; Lyra, M. R.; Pinto Jr., V. L.; Cantalice Filho, J. P.; Oliveira, A. L. L.; Carvalho, L. M. A.; Gross, J. B.; Sousa, A. L. S.; Moraes, T. I.; Bermudez-Aza E. H.; Martins, E. B.; Coura, J. R. 2006. Mass treatment for intestinal helminthiasis control in an Amozonian endemic area in Brazil. Revista do Instituto de Medicina Tropical de São Paulo, 48 (4): 189-195.

Buschini, M. L. T.; Pittner, E.; Czervinski, T.; Moraes, I. F.; Moreira, M. M.; Sanches, H. F.; Monteiro, M. C. 2007. Spatial distribution of enteroparasites among school children from Guarapuava, State of Paraná, Brazil. Revista Brasileira de Epidemiologia, 10 (4): 568-578

Castro, A. Z.; Viana, J. D. C.; Penedo, A. A.; Donatele, D. M. 2004. Levantamento das parasitoses intestinais em escolares da rede pública na cidade de Cachoeira de Itapemirim - ES. NewsLab, 64: 140-144.

FATMA - Fundação do Meio Ambiente. 2006. Banco de dados agregados. Destaques: Balneabilidade. Disponível em $<$ http:// www.fatma.sc.gov.br>. Acesso em 01 abril de 2006.

Faust, E. C.; Sawitz, W.; Tobie, J.; Odom, V.; Peres, C.; Lincicome, D. R. 1939. Comparative efficiency of various technics for the diagnosis of protozoa and helminth in feces. Journal of Parasitology, 25: 241-262.

Ferreira, J. R.; Volpato, F.; Carricondo, F. M.; Martinichen, J. C.; Lenartovicz, V. 2004. Diagnóstico e prevenção de parasitoses no reassentamento São Francisco em Cascavel - PR. Revista Brasileira de Análises Clinicas, 36 (3): 145-146.

Ferreira, P.; Lima, M. R.; Oliveira, F. B.; Pereira, M. L. M.; Ramos, L. B. M.; Marçal, M. das G.; Costa-Cruz, J. M. 2003. Ocorrência de parasitas e comensais intestinais em crianças de escola localizada em assentamento de sem-terras em Campo florido, Minas Gerais, Brasil. Revista da Sociedade Brasileira de Medicina Tropical, 36 (1): 109-111.

Hoffman W. A.; Pons J. A.; Janer J. L. 1934. The sedimentationconcentration method in schistosomiasis mansoni. Puerto Rico Journal of Public Health and Tropical Medicine, 9: 283-291.

Nolla, A. C.; Cantos, G. N. 2005. Relação entre ocorrência de enteroparasitoses em manipuladores de alimentos e aspectos epidemiológicos em Florianópolis, Santa Catarina, Brasil. Cadernos de Saúde Pública, 21 (2) : 641-645.

Quadros, R. M. de; Marques, S.; Arruda, A. A. R.; Delfes, P. S. W. R.; Medeiros, I. A. A. 2004. Parasitoses intestinais em centros de educação infantil municipal de Lages, SC, Brasil. Revista da Sociedade Brasileira de Medicina Tropical, 37 (5): 422-423.

Saturnino, A. C. R. D.; Nunes, J. F. de L.; Silva, E. M. de A. 2003. Relação entre a ocorrência de parasitas intestinais e sintomatolo- 
gia observada em crianças de uma comunidade carente de Cidade Nova, em Natal - Rio Grande do Norte, Brasil. Revista Brasileira de Análises Clínicas, 35 (2): 85-87.

Silva, C. G. da; Santos, H. A. dos. 2001. Ocorrência de parasitoses intestinais da área de abrangência do Centro de Saúde Cícero Idelfonso da Regional Oeste da Prefeitura Municipal de Belo Horizonte, Minas Gerais. Revista de Biologia e Ciências da Terra, 1 (1): 32-43.

Teixeira, J. C.; Heller, L.; Barreto, M. L. 2007. Giardia duodenalis infection: risk factors for children living in sub-standard settlements in Brazil. Cadernos de Saúde Pública, 23 (6):1489-1493.
UNEP. 2006. Challenges to International Waters - Regional Assessments in a Global Perspective. United Nations Environmental Programme, Nairobi, Kenya, 120pp.

Vieira, A. S; Bernardini, O. J.; Ishida, M. M. I. 2005. Pesquisa de enteroparasitas humanos em área de mangue contaminada por esgoto doméstico, na Cachoeira do Bom Jesus, Ilha de Santa Catarina, Florianópolis (SC), Brasil. Anais da $5^{\text {a }}$ Semana de Ensino, Pesquisa e Extensão da Universidade Federal de Santa Catarina, Florianópolis, Brasil, CD Rom. 